\title{
LA TECNOLOGÍA EN LAS RELACIONES CTS. UNA APROXIMACIÓN AL TEMA
}

\author{
ACEVEDO DÍAZ, J.A. \\ Servicio de Inspección. Delegación Provincial de Educación y Ciencia. \\ Alameda Sundheim, 17. Huelva
}

\begin{abstract}
SUMMARY
The aim of this paper is to revalue the technology in STS perspective. First, being based on Kline's typology and Pacey's conceptual model, several meanings of the technology are described. Second, the technological literary meaning is focused on the citizen training to participate in the democratic process of the sociotechnological decision making. Finally, after a review of the principal results obtained in the students' and teachers' beliefs research about the nature of technology and science, and their interactions within society, we tentatively make a STS proposal for the technology education in the compulsory secondary school.
\end{abstract}

\section{INTRODUCCIÓN}

Vivimos, sin duda, en una sociedad tecnificada que aún se impreganará con más técnica, se dice, en el siglo venidero. En efecto, la tecnología forma parte de diferentes aspectos de nuestras vidas desde que nacemos hasta que morimos. Por su parte, la sociedad origina algunas demandas tecnológicas, supuestamente para mejorar la calidad de vida, pero también intenta controlar la tecnología a través de la política y de los medios legales disponibles. A la vez, la tecnología influye en los ciudadanos en la medida en que hacen uso de ella. Como resultado de la aceptación social, o de la imposición más o menos sutil, de determinadas tecnologías en vez de otras, la sociedad también cambia profundamente, siendo moldeada por la tecnología.

Consecuentemente, en los últimos años diversos países, entre ellos España, han introducido, o lo vienen haciendo, la formación tecnológica en los currículos de la enseñanza general obligatoria (UNESCO, 1983). Para ello se han esgrimido razones económicas, sociales y educativas (Gilbert, 1992; Medway, 1989). En diversas ocasiones la Division of Science, Technical and Environmental Education de la UNESCO le ha dedicado especial atención al tema durante la década de los ochen. ta, desde la perspectiva de lo que se ha dado en denominar «alfabetización» tecnológica para la formación de tos ciudadanos, publicando varios documentos sobre aspectos diversos de la educación tecnológica, tales como su interés social (UNESCO, 1986), su conexión con la ciencia y la sociedad (UNESCO, 1988a, 1988b, 1990) e, incluso, la cuestión del género en relación con programas para estimular a las alumnas hacia el estudio de la ciencia y la tecnología (UNESCO, 1984).

No obstante, a pesar de la vigencia actual de la tecnología su estatus culturaj y académico es notablemente inferior al de la ciencia. Tradicionalmente la técnica ha sido marginada por la filosofía en beneficio de la ciencia, de la ciencia «pura» deberíamos precisar, si nos atenemos a esa distinción filosofica de la ciencia moderna en «pura» y «aplicada». Como ha señalado con acierto Medina (1989), el desinterés filosófico por la técnica se debe, sobre todo, al dogma cultural que prima el conocimiento teórico frente al saber ligado a tas capacidades operativas propias de las técnicas; supremacía racional y dogmática de la teoría, que nace con el programa teoricista desarrollado por la antigua filosofía helenística y que se extiende desde to epistemológico hasta io ético y lo político (Luján, 1989; Medina, 1988).

Este dogma teoricista, presente siempre en la civilización occidental, condujo a finales del siglo XIX a que la ciencia moderna se apropiara en gran parte de la tecnología, subordinándola a to abstracto y considerándola, 
ría aquélla capacitada para informarse y reflexionar sobre cuestiones importantes de la sociotecnología, tales como:

- Las ideas sobre el progreso tecnologico.

- Qué tecnologías pueden resultar adecuadas.

- Los beneficios y costes del desarrollo tecnológico.

- Los modelos económicos ligados a la tecrología.

- Las decisiones personales implicadas en el consumo de productos tecnológicos.

- Cómo se configuran las aplicaciones tecnológicas mediante las decisiones tomadas por aquéllos que gestionan la tecnología.

No obstante su interés e importancia, hay que ser conscientes de las complicaciones que puede presentar este enfoque de la «alfabetización» tecnologica a la hora de llevarlo a la práctica con estudiantes de enseñanza secundaria obligatoria, dado que éstos suelen tener bastante desconocimiento de los numerosos factores sociales que, conjuntamente con otros emocionales y psicológicos, afectan a la toma de decisiones sobre tecnología y socicdad (Aikenhead, 1985). Al mismo tiempo una visión más o menos distorsionada de las características, limitaciones y posibilidades de la tecnología, así como la ignorancia de ciertos valores constitutivos y contextuales de la técnica (Layton, I988), igualmente necesarios para el debate, pueden hacer mucho más difícil su ejecucion en el aula. En tal caso, resulta imprescindible conocer las concepciones, creencias y opiniones sobre estas cuestiones del alumnado y del propio profesorado, para poder incorporarlas explícitamente en los procesos de aprendizaje y enseñanza de toma de decisiones sociotecnológicas.

\section{LA TECNOLOGÍA EN LA INVESTIGACIÓN DE LAS CONCEPCIONES CTS}

Posiblemente el instrumento más desarrollado hoy en día en la investigación de las concepciones, creencias y opiniones sobre las relaciones entre ciencia, tecnología y sociedad es el Views on Science-Technology-Society (VOSTS), elaborado por Aikenhead y otros (1987). Desde sus primeras versiones ha incluido cuestiones acerca del papel de la tecnología en las interacciones CTS (Aikenhead et al., 1987; Fleming, 1987), habiéndose centrado los aspectos abordados en:

- Las relaciones entre ciencia y tecnología (diferencias y conexiones entre ambas).

- La influencia de la tecnología en la sociedad (su papel en la resolución de problemas sociales; tecnocracia y democracia en la toma de decisiones socio-tecnologicas).
- La influencia de la sociedad en el desarrollo tecnológico (control social de la tecnología, efectos del ambiente político de un país sobre la tecnología).

Posteriormente, siempre refiriéndonos a la tecnología, se han ampliado estas cuestiones incluyendo (Aikenhead et al., 1989; Aikenhead y Ryan, 1992).

- Diferentes acepciones de la noción de tecnología.

- Los significados de investigación y desarrollo tecnológico $(\mathrm{I}+\mathrm{D})$.

- Más aspectos de la influencia de la tecnología en la sociedad (responsabilidad social de los tecnólogos en relación con los efectos negativos de los impactos tecnológicos; contribución de la tecnología al poder militar, a la economía y al pensamiento social).

- Más aspectos de la influencia de la socicdad en el desarrollo tecnológico (de la política y el gobierno; de la industria civil y militar; de las instituciones educativas; de las asociaciones no gubernamentales y los grupos de presión: consumidores, ecologistas, pacifistas, etc.; la ética en la tecnología).

- Construcción social de la tecnología (autonomía tecnológica; influencias nacionales en las decisiones tecnológicas).

Como señalan Aikenhead y Ryan (1992), el VOSTS es un instrumento abierto a la incorporación de nuevos temas de interés para los estudios CTS.

Por su parte, recientemente, Rubba y Harkness (1993) han observado que en muchas de las preguntas del VOSTS no se separan la ciencia y la tecnología para analizar sus relaciones con la sociedad. Para subsanar esta cuestión han elaborado un instrumento alternativo, destinado preferentemente a investigar las opiniones del profesorado, que denominan Teachers' Beliefs about Science-Technology-Society (TBA-STS). En la primera versión presentada, el instrumento cuenta con trece f́tems, de los que seis hacen referencia a la tecnología:

- ¿Qué es la tecnología?

- ¿Cómo afecta la ciencia a la tecnología?

- ¿Cómo afecta la tecnología a la ciencia?

- ¿Cómo afecta la sociedad a la tecnología?

- ¿Cómo afecta la tecnología a la sociedad?

- ¿En qué sentido fluyen las influencias en las interacciones CTS?

En nuestra opinión el TBA-STS, aunque mucho menos completo que el VOSTS, es más directo y sencillo, por lo que podría resultar adecuado para la utilización en estudiantes de enseñanza secundaria (bachillerato y formación profesional).

A continuación resumiremos los principales resultados de algunas de las investigaciones más recientes, realizadas en Norteamérica (Canadá y Estados Unidos), que han utilizado los instrumentos citados con muestras 
que incluyen desde estudiantes de enseñanza secundaria superior hasta profesores en activo.

Según sefiala Fleming (1987), cuando se les pregunta en términos generales, los estudiantes parecen distinguir los papeles propios que juegan la ciencia y la tecnologia (Price, 1972), siendo capaces de reconocer también las relaciones entre ambas. Sin embargo, matiza que al profundizarse con otras preguntas del VOSTS se comprueba que esto no es así y que, quizás como consecuencia de una interdependencia mal entendida entre ambas, la ciencia y la tecnología se identifican como una empresa única que afecta Ia sociedad: la «tecnociencia». Rubba y Harkness (1993) hacen referencia también a la misma idea, apuntando que tampoco los profesores son capaces de distinguir adecuadamente entre las dos. Esta concepción de «tecnociencia» tiene sus repercusiones a la hora de entender Ia política $\mathrm{I}+\mathrm{D}$ de investigación científica y desarrollo tecnológico (Fleming, 1989).

Ligada a lo anterior aparece la cuestion de la naturaleza específica del conocimiento tecnológico frente a la concepción de la tecnología como ciencia aplicada (Fleming, 1989; Kline, 1985; Luján, 1989; Medina, 1989; Sanmartín, 1990b), ilustrada con rotundidad en el lema que abría la Guía de la Exposición Universal de Chicago (1933): «La ciencia descubre, el genio inventa, la industria aptica y el hombre se adapta o es moldeado por las cosas nuevas..." (citado por Sanmartín, 1990a, p. 168). Esta idea de que la tecnología no es más que la aplicación de la ciencia a la vida cotidiana aparece claramente reflejada en las respuestas de más de la mitad del profesorado encuestado por Rubba y Harkness (1993).

Otros aspectos investigados tratan acerca del cortrol social de la ciencia y la tecnología, así como sobre quién debe tomar las decisiones en las cuestiones de interés sociotecnologico. Más de la mitad de los estudiantes graduados en enseñanza secundaria superior (Fleming, 1987) y una mayoría de los estudiantes universitarios de ciencias (Fleming 1988) se muestran proclives al modelo tecnocrático que propugna que las decisiones importantes en cuestiones sociales relacionadas con las implicaciones tecnológicas deben ser tomadas por una élite de expertos, científicos e ingenieros.

Por otro lado, aunque también se ha encontrado un cierto apoyo al control externo de la tecnología, la mayoría considera que el gobierno es el que está más capacitado para coordinar los programas de investigación científica y desarrollo tecnológico a través de sus agencias especializadas (Fleming, 1987), lo que en nuestra opinión supone también la adopción de una posición favorable a un modelo político tecnocrático.

En resumen, todos estos resultados muestran que la mayor parte de los estudiantes, y probablemente muchos profesores, contemplan las complejas interacciones CTS en las que se ve envuelta la tecnología desde una perspectiva más bien simplista (Rennie, 1987).

Conviene tener en cuenta también que, con frecuencia, las respuestas a cuestiones CTS del tipo de las propues- tas en el VOSTS están ligadas al contexto porque las percepciones de las personas sobre estos temas suelen depender en buena medida de normas sociales, culturales y políticas de carácter local. Por tal motivo, algunas de las creencias que acabamos de exponer sólo pueden tomarse como tendencias de opinión que habría que contrastar en otros sistemas educativos distintos, y que además deberían confirmarse utilizando metodologías e instrumentos de investigación diversos.

Que tengamos constancia (a través de revistas especializadas, congresos, simposios, etc.), son muy pocos los trabajos de investigación en enseñanza con una orientación CTS realizados en España, y casi todos los que conocemos se han hecho desde la perspectiva de la didáctica de las ciencias (Solbes y Vilches, 1989, 1992; Vilches, 1993, 1994), algunos de los cuales hemos comentado en otro lugar (Acevedo, 1994b), sobre todo aquéllos que tocan determinados aspectos ligados a la educación tecnológica: intereses y actitudes hacia la tecnología, integración de la tecnología en la enseñanza de la física y química... Obviamente, aún son menos los que tratan sobre el tema que estamos abordando aquí acerca de las creencias y las opiniones sobre la tecnología en las interacciones CTS. Los resultados que hemos obtenido al analizar las respuestas de estudiantes de BUP y COU (Acevedo, 1992, 1993) y de graduados o licenciados universitarios en ingeniería o ciencias que realizaban el CAP (Acevedo 1994a) a este tipo de cuestiones y otras de CTS relacionadas con la ciencia, similares a las del VOSTS, confirman las tendencias de opinión señaladas, aunque con ciertos matices en algunos casos.

Pese a la manifestada escasez de trabajos de investigación sobre la enseñanza CTS realizados en el contexto de nuestro sistema educativo, hay indicios de que la situación podría estar cambiando y de que, en un futuro próximo, el número de artículos y publicaciones sobre el tema CTS se va a incrementar notablemente en nuestro país, configurándose como un campo de investigación e innovación educativa muy prometedor para la enseñanza de las ciencias y, así lo esperamos con optimismo, para la enseñanza de la tecnología.

\section{UN ENFOQUE CTS PARA LA EDUCACIÓN TECNOLOGICA EN LA ENSENANZA OBLIGATORIA}

Numerosos informes de política educativa de diversos países, sobre todo del ámbito cultural occidental, hacen hincapié en la necesidad de unos aprendizajes básicos sobre tecnología para todos los estudiantes con el fin de obtener nuevos conocimientos y habilidades para adaptarse al ambiente altamente tecrificado en que vivimos (Waks, 1986). Al mismo tiempo se suele añadir, de acuerdo con las ideas del movimiento CTS, que el estudio de la tecnología debería estar conectado al de la ciencia y al de sus consecuencias sociales. 
En España, para la etapa de la educación secundaria obligatoria (ESO) se ha establecido un área de tecnología diferenciada del área de ciencias de la naturaleza. Sin embargo, las expericncias realizadas en otros países, particularmente de la cultura occidental anglosajona, muestran numerosas dificultades a la hora de introducir la tecnología en el currículo escolar obligatorio. Allsop y Woolnough (1990) han señalado varias de ellas relacionadas principalmente con recursos humanos y materiales de todo tipo, las cuales hemos sintetizado en otro trabajo (Acevedo, 1994b). Además de estas razones, Lewis (1991) hace notar que: «La introducción de la tecnología en los planes de estudios modernos es problemática, no porque se considere poco importante sino porque [...] se requiere dotar al área de una estructura conceptual que permita articularla plenamente» (pp. 152-153). Al mismo tiempo, relaciona las dificultades de los responsables del desarrollo del currículo de tecnología y las del profesorado que tiene que ponerlo en práctica, originadas por la ausencia de un conjunto ordenado de conceptos y de una estructura conceptual capaz de dar sentido a un cuerpo de conocimientos propios, con los problemas de los filósofos para considerar la fílosofía de la tecnología en términos epistemológicos; problemas debidos en buena parte a los motivos que hemos indicado en la introducción de este artículo y a que la filosofía de la tecnología no haya alcanzado aún su madurez pese a los esfuerzos que se vienen realizado en la segunda mitad del siglo $\mathrm{XX}$ (Mitcham, 1989).

Por su propia naturaleza, la tecnología, como área de conocimientos, no puede considerarse como un conjunto acotado, ya que, en un sentido amplio, podría decirse que existen tantas tecnologias específicas como tipos de problemas a resolver, por lo que no resulta fácil ponerse de acuerdo sobre cuáles deben ser los contenidos particulares en torno a las cuales elaborar un desarrollo curricular para la tecnología general. Ahora bien, la tecnología se nutre tanto de conocimientos de otras áreas del saber como de su propia experiencia, construyendo un conocimiento básico estructurado por la tensión entre las demandas de la funcionalidad del diseño o proyecto $y$ las restricciones que impone el entorno sociocultural y el medio ambiente; conocimiento que está orientado hacja una praxis concreta a través de la resolución de problemas y la toma de decisiones. Todo esto se revela claramente en los diversos componentes de la tecnología, entre los que destacaremos los siguientes:

- Componente cientifico-tecnológico. Realza las relaciones mutuas entre ciencia y tecnología respetando sus finalidades y objetivos propios. La tecnología utiliza numerosos conceptos científicos, los cuales sufren un tratamiento previo de reelaboración y reconstrucción conceptual con el fin de integrarlos y adaptarlos para su adecuación al contexto tecnológico. Tambiên hace uso de algunos procedimientos metodológicos simitares a los empleados por la ciencia. Asimismo, ésta también recibe muchas aportaciones de la tecnología, no sólo de los instrumentos y sistemas que produce sino de los conocimientos teóricos y metodológicos que elabora.
- Componente histórico-cultural. Subraya la relación entre las técnicas desarrolladas por la humanidad y los cambios que éstas provocan en el medio natural, en la cultura y en las condiciones de vida de las personas. Igualmente se ocupa de cómo la sociedad condiciona la actividad tecnológica. En este componente se incluyen técnicas artísticas como la arquitectura, la pintura, la escultura, la música, la fotografía, cl cine, etc.

- Componente organizativo-social. Da relevancia a la tecnología como factor que influye decisivamente sobre las formas de organización social. A la vez muestra cómo la dimensión organizativa es uno de ios etementos importantes de la trama tecnológica.

- Componente verbal-iconográfico. Muestra las diversas formas de expresión y comunicación propias de la tecnología: simbolos, vocabutario específico, ete.

- Componente técnico-metodológico. Entendido como el conjunto de capacidades y destrezas técnicas necesarias para manipujar los instrumentos y fabricar los productos tecnológicos, así como los procedimientos y estrategias que hacen falta para resolver problemas reales en situaciones concretas.

Como consecuencia de los argumentos anteriores, no resulta difícil entender que se hayan planteado diversas opciones, no necesariamente incompatibles entre sí, para la educación tecnológica en la enseñanza obligatoria. Así, basándose en que la tecnología refleja distintos aspectos de la vida humana y en que utiliza conceptos procedentes de diversas áreas de conocimiento, en algunas ocasiones se ha llegado a proponer el tratamiento transversal de la tecnología en el plan de cstudios (Woolnough, 1975). La elección de este enfoque tiene cierto sentido por la presencia de lo tecnológico en lo científico, lo social, lo artístico, etc., pero también se corre el riesgo de que la tecnología quede difuminada y no alcance suficiente relevancia porque el profesorado presta una atención preferente al área o las materias de su especialidad.

Otra opción, cuya práctica es más frecuente en la enseñanza, tanto dentro como fuera del marco CrS , es la de la integración de la tecnología en las disciplinas del área de ciencias (Acevedo, 1990; Allsop y Woolnough, 1990; Casalderrey, 1986; Fensham, 1988; FernándezUría, 1979; Gil, 1994b; Gilbert, 1992; Layton, 1988; Woolnough, 1988), sobre todo en la física y la guímica. Sin embargo, puesto que esta modalidad de enseñanza se encuentra mayoritariamente en manos de un profesorado con formación universitaria en ciencias académicas $y$, generalmente, con poca experiencia en tecnología, la misma ha contribuido a menudo a reforzar una visión jerarquizada en la que la tecnología se contempla como una mera aplicación de la ciencia, supeditándose à ésta (Acevedo, 1994b)

El tercer punto de vista, que se apoya en que la cducación tecnologica debe atender objetivos específicos diferentes a los de la formación científica, la social y la artística, aunque se relacione con ellas, supone la apues- 
ta por su tratamiento disciplinar o a nivel de área, tal y como ocurre en Ia ESO, en el sistema educativo español. De nuevo debido a la formación, esta vez básicamente de carácter técnico, del profesorado que imparte la materia, también se corre el riesgo de transmitir, a través del currículo, un punto de vista acumulativo y lineal del desarrollo tecnológico por el que el progreso social se confunde con los cambios técnicos producidos siguiendo el criterio de una mayor eficiencia; sin duda, una perspectiva sesgada que se basa en la tradición más dura de la ingeniería y que pretende legitimar, entre otras cosas, el imperativo y el determinismo tecnológico.

Centrándose en las finalidades y objetivos de la educación tecnológica en la enseñanza obligatoria, Zoller y Watson (1974) han resaltado algunos de los aspectos que aeben caracterizarla, entre ellos:

- Dirigir los contenidos hacia problemas reales en su contexto social.

- Dar más importancia, a expensas de lo teórico, a las cuestiones prácticas.

- Considerar las consecuencias de los impactos tecnológicos en la sociedad.

- Tener en cuenta no sólo las posibilidades de la tecnología para la resolución de problemas sociotécnicos, sino también sus limitaciones.

Para Fleming (1989), la visión sociotecnológica de la tecnología es la más apropiada, por lo que las instituciones educativas deberían preparar a los estudiantes de todos los niveles, y más concretamente en la educación secundaria obligatoria, para su participación pública en una efectiva toma de decisiones en un mundo sociotecnológico. Como ya hemos señalado en su momento, ésta es la principal finalidad de la forma más democrática de entender la «ałfabetización» tecnológica desde un punto de vista CTS (Waks, 1990).

Por otro lado, partiendo del modelo conceptual sobre la tecnología y su práctica, propuesto por Pacey (1983), al quc hemos aludido en este artículo, Gilbert (1992) ha hecho un análisis sobre las diferentes formas de abordar la educación tecnológica según qué aspectos se atiendan en tos contenidos. En su estudio, que incluye numerosos elementos didácticos para la reflexión curricular acerca de la enseñanza de la tecnología, Gilbert distingue entre:

- Formación para la tecnología (centrada en los aspectos técnicos del modelo de Pacey). Ésta es la perspectiva más habitual en la enseñanza, pero también la más restringida.

- Formación sobre la tecnología (dirigida sobre todo a las cuestiones sociotecnológicas del modelo, esto es, a las organizativas e ideológicas). Ésta es la visión más frecuente en los enfoques CTS que subrayan los aspectos sociales de la tecnología.
- Formación en la tecnología (que toma en cuenta las tres dimensiones consideradas por Pacey). Según Gilbert, adoptar esta elección supone decidirse por una enseñanza comprensiva y holística de la tecnología.

A nuestro juicio, desde una perspectiva CTS 10 más amplia posible, una propuesta de educación tecnológica centrada en la enseñanza secundaria obligatoria (12-16 años de edad) debería contemplar al menos los siguientes aspectos:

- Dar un enfoque constructivista al aprendizaje y la enseñanza de la tecnología que tenga en cuenta las concepciones, los intereses y las actitudes de las alumnas y los alumnos, y que sea capaz de superar también las diferencias de género atendiendo a la diversidad (Kelly, 1988; Rennie, 1987).

- Abordar en la enseñanza aquellos problemas sociotecnológicos prácticos que sean verdaderamente relevantes para el alumnado, tanto los que pueden contribuir a resolver la tecnología como aquéllos que origina (Accvedo, 1992). Estos problemas podrán ser históricos, contemporáneos o futuros, aunque probablemente sean los actuales los más adecuados por ser más reales y tangibles.

- Aprovechar el tratamiento de los problemas sociotécnicos para situar en contextos específicos los conceptos de la ciencia que utiliza la tecnología, rebajando así su nivel de abstracción (Layton, 1988).

- Utilizar los problemas sociotecnológicos con los que se trabaje para introducir algunos aspectos sociales relacionados con la tecnología: filosóficos, sociológicos, éticos, políticos, económicos, valores propios de la técnica, etc. (Rosenthal, 1989).

- Contribuir a desarrollar las capacidades necesarias para argumentar las opiniones en relación con la toma de decisiones sobre cuestiones CTS en una sociedad democrática (Aikenhead, I985; Waks, 1990).

- Favorecer la apertura del ámbito escolar al medio social en que se encuentra (Penick y Yager, I986); por ejemplo, promoviendo la participación en el aula de especialistas, que pueden ser los mismos padres o madres de los estudiantes; mediante la visita a fábricas, museos de la técnica y complejos de interés tecnológico; extendiendo la formación a las empresas y centros de trabajo; etc.

Somos conscientes de que una propuesta como ésta supone un serio esfuerzo para la administración educativa, para el profesorado y para la sociedad en general, ya que implica: mayor atención a los recursos materiales (instalaciones adecuadas y nuevos medios didácticos) y humanos (más y mejor formación del profesorado), introducir nuevas formas organizativas en los centros, un profundo trabajo de planificación y diseño curricular, etc. Pero, en definitiva, se trata de revalorizar la tecnología dentro del marco que promueve el movimiento CTS, tal y como se expresa explícitamente con acierto en 
cl título de un artículo de Layton (1988). Todo un reto social, sin duda, para mejorar la educación tecnológica en la enseñanza obligatoria y para darle, de una vez por todas, el lugar que le corresponde en la cultura del mundo actual.

\section{A MODO DE CONCLUSIÓN: ALGUNAS REFLEXIONES FINALES PARA ORIENTAR LA CONSTRUCCION DE UNA FUTURA DIDACTICA DE LA TECNOLOGIA}

En un interesante artículo de recapitulación sobre las investigaciones llevadas a cabo en el campo de la didáctica de las ciencias en los útimos años, Gil (1994a) recuerda que, aunque el germen estaba puesto (Fernández-Uría, 1979), a comienzos de los años ochenta todavía se hablaba de la didáctica de las ciencias como un dominio de conocimientos en estado preteórico. Desde entonces se ha visto sometida a enormes transformaciones, empezando a mostrarse en la actualidad como un cuerpo coherente de conocimientos en construcción (Aliberas et al., 1989; Furió y Gil, 1989; Gil et al., 1991), capaz de crecer independientemente de otros saberes con los que se relaciona (Gil, 1993a,b) como consecuencia del impresionante incremento cuantitativo y, sobre todo, cualitativo experimentado por la investigación en este campo. Todo esto está teniendo además sus efectos en las correspondientes orientaciones para la formación del profesorado de ciencias (Furió, 1994; Gil, 1991).

En cambio, está aún pendiente de definir claramente el camino que se va a tomar para la elaboración de una didáctica de la tecnología, no sólo en España, donde la disciplina es casi una recién nacida, sino también en otros países con mucha más tradición que el nuestro en la enseñanza de la tecnología, habiéndose apuntado en este artículo algunas de las razones que justifican esta situación. En relación con este problema, Gilbert (1992) propugna unas relaciones más efectivas que las que se han venido dando hasta ahora entre ambas enseñanzas, formulando un listado de dieciséis preguntas que podrían resultar relevantes para la investigación en la enseñanza de la tecnología, de las que destacaremos, a título de ejemplo, las siguientes:

- ¿Cuál debe ser la estructura disciplinar de la enseñanza de la tecnología?

- ¿Qué repercusiones tiene la enseñanza de la tecnología en las actitudes hacia la tecnología y en la formación integral del alumnado?

- ¿Cómo preparar al profesorado para poder proporcionar una educación «en» tecnología?

- ¿Qué aportaciones podría hacer la didáctica de las ciencias a las modalidades de enseñanza "para», "sobre» $y$ «en» la tecnología?

Creemos que cuestiones como las planteadas por Gilbert son importantes para el desarrollo de una didáctica de la tecnología y que, asimismo, las relaciones entre la enseñanza de las ciencias y la de la tecnología no están suficientemente exploradas a nivel escolar.

Por otro lado, en el Diseño Curricular Base (DCB) de la ESO (MEC, 1989) se indica, para el area de Ciencias de la Naturaleza, que: «En la medida en que nuestra sociedad está fuertemente impregnada de elementos científicos y tecnológicos, a través de esta área los alumnos son capacitados para poder participar activa y críticamente en la solución de muchos de los problemas que hoy afronta la sociedad en el uso, disfrute y conservación de la naturaleza» (p. 84). Y, más adelanté, para el área de Tecnología, que eincrementa la funcionalidad de los saberes adquiridos a través de la resolución de problemas prácticos reales y la toma de decisiones ante situaciones concretas. Por otra parte, desarrolla capacidades đe inserción y actuación social...» (p. 85). Ambos párrafos contienen, en nuestra opinion, aspectos educatives claramente asumidos por el movimiento de enseñanza CTS, tal y como se desprende, por ejemplo, de las conclusiones de los debates del IV Simposio Intemacional sobre Tendencias Mundiales de Educarión en Ciencia y Tecnología (Hofstein et al., 1988), organizado por la International Organization for Science and Technology Education (IOSTE) y realizado en agosto de 1987 en el IPN de Kiel (Alemania), donde se afirmó con rotundidad que la educación CTS tiene que formar parte de todos los cursos de ciencia y tecnología, requiriéndose para ello, además de una visión más holística de ambas áreas de conocimientos, serios esfuerzos destinados a:

- Clarificar el significado de la perspectiva CTS en las enseñanzas de la ciencia y la tecnología.

- Desarrollar materiales curriculares CTS de todo tipo.

- Formar al profesorado en el marco de la enseñanza CTS.

- Elaborar estrategias eficaces para la enseñanza y el aprendizaje CTS.

- Implicar a responsables de la administración y de la comunidad educativa en el apoyo a lá educación CTS.

- Establecer normas y preparar instrumentos y estrategias de evaluación útiles en el contexto de Ia enseñanza CTS.

Por lo tanto, con estas premisas, no debería extrañar a nadie que algunos de los aspectos de la propuesta, que hemos esbozado con anterioridad, para una enseñanza de la tecnología inmersa en la perspectiva CTS pueden guardar cierta semejanza con los procedentes de otras que se están haciendo en la didáctica de las ciencias. Fà efecto, sin olvidar que muchos de los contenidos de los proyectos CTS que se vienen incorporando a la enseñanza de las ciencias corresponden en mayor medida al dominio de lo tecnologico que al estrictamente científico (comparar, por ejemplo, los temas CTS señalados por Bybee, 1987, con los de tecnología indicados por 
Lewis, 1991), según nuestro punto de vista es precisamente la aproximación CTS de la enseñanza la que mejor podría contribuir, por su carácter más global, a investigar qué relaciones deben darse entre las enseñan-

\section{REFERENCIAS BIBLIOGRÁFICAS}

ACEVEDO, J.A. (1990). Estudios de casos de innovación: enseñanza de la física en contexto. Investigación en la Escuela, 12, pp. $91-92$.

ACEVEDO, J.A. (1992). Cuestiones de sociología y epistemología de la ciencia. La opinión de los estudiantes. Revista de Eáucación de la Universidad de Granada, 6, pp. 167-182.

ACEVEDO, J.A. (1993). ¿Qué piensan los estudiantes sobre la ciencia? Un enfoque CTS. Enseñanza de las Ciencias, núm. extra (IV Congreso), pp. 11-12.

ACEVEDO, J.A. (1994a). Los futuros profesores de enseñanza sccundaria ante la sociología y la epistemología de las ciencias. Revista Interuniversitaria de Formación del Profesorado, 19, pp. 111-125.

ACEVEDO, J.A. (1994b). Educación tecnológica desde una perspectiva CTS. Una breve revisión del tema. Alambique, 3 (aceptada su publicación).

AIKENHEAD, G.S. (1985). Collective decision making in the social context of science. Science Education, 69(4), pp. 453. 475.

AIKENHEAD, G.S., FLEMING, R.W. y RYAN, A.G. (1987). High-school graduates' beliefs about science-technologysociety. I. Methods and issues in monitoring student views. Science Education, 71(2), pp. 145-161.

AIKENHEAD, G.S., RYAN, A.G. y FLEMING, R.W. (1989). Views on science-technology-society (form CDN. mc. 5). Saskatoon, Canadá: Department of Curriculum Studies. University of Saskatchewan, S7N OW0.

AIKENHEAD, G.S. y RYAN, A.G. (1992). The development of a new instrument: «Views on Science-Technology-Society» (VOSTS). Science Education, 76(5), pp. 477-491.

ALIBEIRAS, J., GUTIÉRREZ, R. e IZQUIERDO, M. (1989). La didàctica de les ciències: una empresa racional. Enseñanza de las Ciencias, 7(3), pp. 277-284.

ALLSOP, R.T. y WOOLNOUGH, B.E. (1990). The relationship of technology to science in English schools. Journal of Curriculum Studies, 22(2), pp. 127-136.

AMERICAN ASSOCIATION FOR THE ADVANCEMENT OF SCIENCE (1989). Project 2061: Science for all Americans. Washington D.C.: AAAS.

BASALLA, G. (1988). The evolution of technology. Cambridge: Cambridge University Press. Traducción de J. Vigil (1991). La evolución de la tecnología. Barcelona: Crítica.

BERNAL, J.D. (1964). Science in History. (Watts: Londres). Traducción de Capella, J.R. (1967). Historia social de la ciencia. Barcelona: Penínsuia.

BYBEE, R.W. (1987). Science education and the ScienceTechnology-Society (S-T-S) theme. Science Education, 71(5), pp. 667-683.

CASALDERREY, M.L. (1986). Aproximación a la integración ciencia-tecnologia. Madrid: Servicio de Publicaciones del MEC.

CHALMERS, A. F. (1990). Science and its fabrication. Milton Keynes: Open University Press. Traducción de Pérez Sedeño, E. (1992). Laciencia y como se elabora. Madrid: Siglo XXI. zas de la ciencia y de la tecnología en un plano de igualdad, lo que hasta ahora ha resultado ser con frecuencia más bien una buena intención que una práctica habitual.

FENSHAM, P.J. (1988). Approaches to the teaching of STS in Science Education. Intemational Joumal of Science Education, IO(4), pp. 346-356

FERNÁNDEZ-URÍA, E. (1979). Estructura y didactica de las ciencias. Madrid: Servicio de Publicaciones del MEC.

FLEMING, R.W. (1987). High-school graduates' beliefs about science-technology-society. II. The interaction among science, technology, and society. Science Education, 7 l(2), pp. 163 186.

FLEMING, R.W.(1988). Undergraduate science students' views on the relationship between Science, Technology and Socicty. International Journal of Science Education, 10(4), pp. 449. 463.

FLEMING, R.W. (1989). Literacy for a technological age. Science Education, 73(4), pp. 391-404

FURIÓ, C.J. (1994). Tendencias actuales en la formación del profesorado de ciencias. Enseñanza de las Ciencias, 12(2), pp. 188-199.

FURIÓ, C.J. y GIL,D. (1989). La didáctica de las ciencias en la formación inicial del profesorado: una orientación y un programa teóricamente fundamentado. Enseñanza de las Ciencias, 7(3), pp. 257-265.

GIL, D. (1991). ¿Qué hemos de saber y saber hacer los profesores de ciencias? Enseñanza de las Ciencias, $9(1)$, pp. 69-77.

GIL, D. (1993a). Contribución de la historia y de la filosofía de las ciencias al desarrollo de un modelo de enseñanza-aprendizajc como investigacion. Enseñanza de las Ciencias, 11(2), pp. 197-212.

GIL, D. (1993b). Psicología educativa y didáctica de las ciencias: los procesos de enseñanza-aprendizaje de las ciencias como lugar de encuentro. Infancia y Aprendizaje, 62-63, pp. 171185.

GlL, D. (1994a). Diez años de investigación en didáctica de las ciencias: realizaciones y perspectivas. Enseñanza de las Ciencias, 12(2), pp. 154-164.

GIL, D. (1994b). Relaciones entre conocimiento escolar y conocimiento científtco. Investigación en la Escuela, 23, pp. 17-31.

GIL, D., CARRASCOSA, J., FURIÓ, C. y MARTÍNEZ, TORREGROSA, J. (1991). La enseñanza de las ciencias en la educación secundaria. Barcelona: ICE de la Universidad de Barcelona-Horsori.

GILBERT, J.K. (1992). The interface between science education and technology education. Intemational Journal of Science Education, 14(5), pp. 563-578

GÓMEZ, J. e ILERBAIG, J. (1990). Ciencia, tecnología y sociedad. Alternativas educativas para un mundo en crisis, en Medina, M. y Sanmartín, J. (eds.) (1990). Ciencia, tecnología y rociedad, pp. 130-152. Barcelona: Anthropos.

HOFSTEIN, A., AIKENHEAD, G. y RIQUARTS, K. (1988). Discussions over STS at the Fourth IOSTE Symposium. International Journal of Science Education, 10(4), pp. 357366. 
KEI.LY, A. (1988). Option chotce for girls and boys. Research in Science \& Technological Education, 6(1), pp. 5-23.

KLINE, S.J. (1985). What is technology? Bulletin of Science, Technoloy' and Societ', 5(3), pp. 215-218.

I.AYTON, D. (1988). Revaluing the T in STS. International Journal of Science Education, 10(4), pp. 367-378.

LEWIS, T. (1991). Introducing technology into school curricula. Journal of Curriculum Studies, 10(4), pp. 367-378.

I.UIÁN, J.L. (1989). Tecnología, ciencia y sociedad: proceso a la epistemología popular. Anthropos, 94-95, pp. 81-86.

MEC (1989). Diseño Curricular Base. Madrid: Servicio de Publicaciones del MEC.

MEDINA, M. (1988). Extravios racionales. Anthropos, 82-83, pp. 62-69.

MEDINA, M. (1989). Mito de la teoría y filosofía de la tecnología. Anthropos, $94-95$, pp. $35-39$

MEDWAY, P. (1989). Issues in the theory and practice of technology education. Studies in Science Education, 16, pp. $1-24$.

MITCHAM, C. (1989). ¿Qué es la filosofía de la tecnología? Barcelona: Anthropos.

MOKYR, J.(1990). The lever of riches. Technological creativity and economic progress. Oxford: Oxford University Press. Traducción de Gómez Parro, E. (1993). La palanca de la riqueza. Creatividad tecnológica y progreso económico. Madrid: Alianza.

MUMFORD, L. (1934). Technics and civilization. Harcourt: Brace and World. Traducción de Aznar de Acevedo, C. (1971). Técnica y civilización. Madrid: Alianza.

PACFY, A. (1983). The Culture of Technology. Cambridge, MA: MrT Press. Traducción de Ríos, R. (1990), La cultura de la tecnología. México DF: FCE.

PENICK, J.E. y YAGER, R.E. (1986). Trends in Science Education: some observations of exemplary programs in the United States. European Journal of Science Education, $8(1)$, pp. I-9.

PRICE, D.J. DE SOLI.A. (1972). Science and technology: Distinctions and interrelationships, en Barnes, R. (ed.) (1972), Sociology of science. Harmondsworth: Penguin Books, pp. 166-180. Traducción de Miguez, N.A. (1980). Estudios sobre sociología de la ciencia, pp. 163-167. Madrid: Alianza.

RENNIE, L.I. (1987). Teachers' and pupils' perceptions of technology and the implications for curriculum. Research in Science \& Technological Education, 5(2), pp. 121-133.

ROSFNTHAL, D.B. (1989). Two approaches to ScienceTechnology-Society (S-T-S) Education. Science Education, 73(5), pp. $581-589$.

RUBBA, P.A. y HARKNESS, W.L. (1993). Examination of Preservice and ln-Service Secondary Science teachers' beliefs about Science-Technology-Society interactions. Science Education, 77(4), pp. 407-431.

SÁNCHEZ-RON, J.M. (1992). El poder de la ciencia. Madrid: Alianza.

SANMARTÍN, J. (1990a). La ciencia descubre. I.a industria aplica. El hombre se conforma. Imperativo tecnológico y diseño social, en Medina, M. y Sanmartín, J. (eds.) (1990).
Ciencia, tecnología y sociedad, pp. 168-180. Barcelona: Anthropos.

SANMARTÍN, J. (1990b). Tecnologít y futuro humano. Barcelona: Anthropos.

SNOW, R.E. (1987). Core concepts for science and technology literacy. Bulletin of Science, Technology and Socie'ty, $7(5-6)$, pp. $720-729$.

SOLBES, J, y VILCHES, A. (1989). Interacciones cienciatécnica-sociedad: un instrumento de cambio actitudinal. Enseñanza de las Ciencias, 7(1), pp. 14-20.

SOLBES, J. y VILCHES, A. (1992). El modelo constructivista y las rèaciones ciencia-têcnica-sociedad (C'TS). Enseñanza de las Ciencias, 10(2), pp. 181-186.

UNESCO (1983). Technology education as part of general education. Scjence and Technofogy Education Document Series, 4. París: UNESCO.

UNESCO (1984), Encouraging givls into science and technology education: Some Luropean initiatives. Science and Technology Education Documents Series, 7. Paris: UNESCO.

UNESCO (1986). The social relevance of science and technology education. Science and Technology Fducation Document Series, 18. París: UNESCO.

UNESCO (1988a). Pour un enseignement integré de la sciunce et de la technologie: trois modules. Science and Technology Education Document Series, 27. l'arís: LNFSCO.

UNESCO (1988b). Educational materials linking t'chnology teaching with science education: Technology in life. Science and Technology Education Document Series, 31. Paris: UNESCO.

UNESCO (1990). The teaching of science and technology in an interdisciplinary context. Science and Technology Educition Document Series, 38. París: UNESCO.

VILCHES, A. (1993). Las interacciones CTS y la enseñan-a de las ciencias fisico-químicas. Tesis doctoral. Facultad de Física. Universidad de Valencia.

VILCHES, A. (1994). Lá introducción de las interacciones: ciencia, técnica y sociedad (CTS). Una propuesta necesaria en la enseñanza de las ciencias. Atta de Mnos acion Educativa, $27, \mathrm{pp} .32-36$.

WAKS, L.J. (1986). Reflections on technological literacy. Bulletin of Science, Technology, and Socicty, 6 (2-3), pp. 331-336.

WAKS, L.J. (1990). Educación en ciencia, tecnología y socicdad: orígenes, desarrollos internacionales y desaffos actuales, en Medina, M. y Sanmartín, J. (eds.) (1990). Ciencia, techologia $y$ sociedad, pp, 42-75. Barcelona: Anthropos.

WAKS, L.J, y PRAKASH, M.S. (1985). STS education and its three step- sisters. Bulletin of Science. Technology and Society, S(2), pp. 105-116.

WOOLNOUGH, B.E. (1975). The place of technology in schosls. The School Science Review, 56(196), pp. 443-448.

WOOLNOUGH, B.E. (1988). Technological Edacation and Science in Schools. Hatfield, Herts: ASE.

7OL.LER, U. y WATSON, F.G. (1974). Teclmology education for non-science students in the Secondary Schooln. Scicnce Education, 58(1), pp. 105-116. 